\title{
Effectiveness and cost-effectiveness of vaccination against herpes zoster in Canada: a modelling study
}

\author{
Mélanie Drolet PhD, Zhou Zhou MD PhD, Chantal Sauvageau MD MSc, Philippe DeWals MD PhD, \\ Vladimir Gilca PhD, Rachid Amini MSc, Élodie Bénard MSc, Marc Brisson PhD
}

Cite as: CMAJ 2019 August 26;191:E932-9. doi: 10.1503/cmaj.190274

\section{ABSTRACT \\ BACKGROUND: Two vaccines against her- pes zoster are currently authorized for use in Canada: the recombinant subunit zos- ter vaccine and live attenuated zoster vac- cine. We compared the effectiveness and cost-effectiveness of these 2 vaccines.}

METHODS: We used a decision analytic static cohort model parametrized with Canadian epidemiologic and economic data. We performed the economic analysis from the health care system perspective, using a lifetime horizon and a $3 \%$ discount rate for costs and benefits. The primary outcome was the incremental cost per quality-adjusted lifeyear (QALY) gained, relative to no vaccination. We ran 30000 simulations varying all model parameters, including vaccine costs, efficacy and waning.

RESULTS: The number needed to vaccinate (NNV) was higher for the live attenuated zoster vaccine than for the recombinant subunit zoster vaccine for all herpes zoster-related events at all ages. For example, in persons exactly 65 years old, for herpes zoster, median NNV was 21 (90\% uncertainty interval [UI] 13-31) versus 8 (90\% UI 6-18), and for postherpetic neuralgia, NNV was 64 (90\% UI 33-93) versus 31 (90\% UI 23-73). For the recombinant vaccine, the median cost-effectiveness ratios varied between cost-saving and \$25881 per QALY gained for adults aged 50 years or older. For the live vaccine, the cost-effectiveness ratios varied between cost-saving and $\$ 130587$ per QALY gained and were less than $\$ 45000$ per QALY gained only for those 65 to 75 years old. Given its higher efficacy, we estimated that the cost for the complete series of the recombinant vaccine could be $\$ 150$ to $\$ 200$ more than the cost of the live vaccine and still be considered cost-effective.

INTERPRETATION: Our model predicted that the recombinant subunit zoster vaccine is likely cost-effective in Canada for adults 60 years or older, and is likely more cost-effective than live attenuated zoster vaccine. These results have informed updated national and provincial recommendations on herpes zoster vaccination.
H erpes zoster, characterized by dermatomal pain and rash, , affects about 1 of every 3 persons during their lifetime. ${ }^{3-5}$ The most common complication is longlasting debilitating pain, known as postherpetic neuralgia, which occurs in about $8 \%$ to $27 \%$ of individuals with herpes zoster. ${ }^{6-10}$ Given that postherpetic neuralgia has a substantial negative impact on health-related quality of life ${ }^{11}$ and that therapeutic options are only partially effective, ${ }^{12}$ the best option remains the prevention of herpes zoster and thus postherpetic neuralgia. ${ }^{13}$

Two herpes zoster vaccines are currently authorized for use in Canada among adults aged 50 years or older: the recombinant subunit zoster vaccine (Shingrix) and the live attenuated zoster vaccine (Zostavax). The recombinant vaccine was approved recently (October 2017), whereas the live vaccine has been available since 2008. Clinical trials have shown that the recombinant vaccine is highly effective against herpes zoster and postherpetic neuralgia for adults aged 50 years or older (vaccine efficacy against herpes zoster $96.6 \%$ for those $50-59 \mathrm{yr}$ and $97.9 \%$ for those $>70 \mathrm{yr}$ ) with no evidence of waning protection after 4 years. ${ }^{14}$ Recent immunogenicity data also suggest that the immune response is maintained up to 9 years after vaccination. ${ }^{15}$

Conversely, clinical trials and observational data have suggested that the efficacy of the live vaccine against herpes zoster decreases with older age at vaccination (from $65.5 \%$ for those $60-69 \mathrm{yr}$ to $55.4 \%$ for those $\geq 70 \mathrm{yr}^{10}$ ) and wanes with increasing time since vaccination. ${ }^{16-18}$

Although the recombinant vaccine appears to be more effective, particularly among older adults, a 2-dose schedule is recommended, compared with a 1-dose schedule for the live vaccine; this difference has implications for costs and vaccination logistics. Furthermore, although both vaccines have been shown to be safe, a significantly higher proportion of adults vaccinated 
with the recombinant vaccine experienced grade 3 adverse events (e.g., injection-site pain, redness or swelling, myalgia, fatigue, headache), relative to those receiving placebo $(17 \% \mathrm{v}$. $3 \%) .{ }^{14}$ These adverse events could affect completion of the vaccination schedule and vaccine efficacy.

Clinicians and policy-makers in various jurisdictions are currently making recommendations about the choice of herpes zoster vaccine to use and the age cohorts to be vaccinated. The criteria considered in such decisions include cost-effectiveness. The aims of this study were to evaluate the effectiveness and costeffectiveness of vaccinating adults 50 years of age or older against herpes zoster in Canada, using 1 of the 2 currently available vaccines (live attenuated zoster vaccine or recombinant subunit zoster vaccine), relative to the absence of vaccination, and then to compare the 2 vaccines in terms of effectiveness and cost-effectiveness. This work informed the 2018 updated recommendations on the use of herpes zoster vaccines by the National Advisory Committee on Immunization $(\mathrm{NACl})^{19}$ and the Comite d'immunisation du Québec. ${ }^{20}$

\section{Methods}

\section{Model structure}

We used a previously published decision analytic static cohort model. ${ }^{4,21}$ The model structure for the current study was the same as previously published, but we updated all parameter values. Briefly, the model followed a cohort of adults through different phases of herpes zoster (no herpes zoster, herpes zoster, postherpetic neuralgia) (Figure A1 in Appendix 1, available at www. cmaj.ca/lookup/suppl/doi:10.1503/cmaj.190274/-/DC1). The model compared the incidence of herpes zoster and postherpetic neuralgia, mortality rate, use of health care resources (in terms of hospitalization, consultations and length of hospital stay), costs and quality-adjusted life-years (QALYs) lost between vaccinated and unvaccinated cohorts of adults.

\section{Vaccine efficacy parameters}

Vaccine efficacy comprises 2 elements: the degree to which adults are protected shortly after vaccination (initial vaccine efficacy) and the loss of vaccine protection over time (waning of vaccine efficacy). We estimated parameter values for the efficacy of the 2 vaccines by fitting the age-specific annual incidence of herpes zoster predicted by the model with that observed in the vaccination arm of randomized clinical trials, ${ }^{10,14,22,23}$ using 6 different functions of waning efficacy over time (for a formal description of these functions, see Table A1 in Appendix 1). This method, based on previous modelling studies, ${ }^{21,24}$ makes it possible to estimate both short-term vaccine efficacy and waning efficacy, as well as to capture the uncertainty surrounding the long-term efficacy of the 2 vaccines (Figure $A 2$ in Appendix 1).

\section{Epidemiologic and economic parameters}

We updated the epidemiologic and economic parameters used by Brisson and colleagues ${ }^{21}$ through literature reviews and analyses of available data sources (Table 1). . $^{3,10,11,21,25-31}$ More specifically, we updated the epidemiologic parameters and health care resource use associated with herpes zoster through a systematic review of the literature and data extraction from Quebec administrative databases, previously published by Letellier and colleagues. ${ }^{25}$ The parameters presented in Table 1 represent the minimum and maximum values identified in the literature (including the article by Brisson and colleagues ${ }^{21}$ ) and obtained from analysis of Quebec administrative databases. We also updated costs related to herpes zoster and postherpetic neuralgia through a literature review. We identified a recent study conducted in Manitoba that specifically estimated the costs associated with herpes zoster and postherpetic neuralgia; ${ }^{30}$ we used the values from this study as our base values. The costs presented in Table 1 also represent the minimum and maximum values identified in the literature. All costs were adjusted to 2018 Canadian dollars according to the Consumer Price Index. ${ }^{32}$ We varied the cost of a complete vaccination series between $\$ 100$ and $\$ 200$ (including both the vaccine price and administration costs).

We performed the analysis from a health care system perspective, on the basis of discussions with Canadian decision-makers, and therefore did not include indirect costs (e.g., wages lost).

\section{Outcomes}

We estimated the following 3 outcomes: pre-vaccination burden of herpes zoster in Canada, effectiveness of herpes zoster vaccination and cost-effectiveness of herpes zoster vaccination.

For the pre-vaccination burden of herpes zoster, we estimated the yearly number of herpes zoster-related events (cases of herpes zoster, ophthalmic herpes zoster, postherpetic neuralgia, hospital admissions and deaths).

For vaccination effectiveness, we used the number needed to vaccinate (NNV), calculating the NNV values as number of people vaccinated divided by number of herpes zoster-related events prevented over a lifetime.

For the cost-effectiveness of herpes zoster vaccination, we used 2 comparisons: vaccination versus no vaccination and recombinant vaccine versus live vaccine. As the primary outcome, we used the incremental cost per QALY gained of herpes zoster vaccination compared with no vaccination. Although there is no recommended costeffectiveness threshold in Canada, we used a threshold of \$45000 per QALY gained, which corresponds to the gross domestic product per capita (as suggested by the World Health Organization ${ }^{33}$ ).

Because the complete series of the recombinant vaccine ( 2 doses) will likely be more costly than the live vaccine (1 dose), our secondary cost-effectiveness outcome was the additional cost of a complete series of the recombinant vaccine, to obtain an incremental cost-effectiveness ratio under the $\$ 45000$ per QALY gained threshold (v. the live vaccine).

\section{Statistical analysis}

We performed the economic analysis from the health care perspective, used a lifetime time horizon and assumed a 3\% discount rate for both costs and benefits (as traditionally used in Canada when assessing the cost-effectiveness of vaccines).

To illustrate results across different cost-effectiveness thresholds, we produced acceptability curves for the vaccination of adults aged 65 years and for vaccine costs of $\$ 140$ and $\$ 200$. 
Table 1: Epidemiologic, health care resource use and economic parameters

\section{Parameter by age category, yr}

\section{Epidemiologic}

Herpes zoster incidence, per 1000 person-years

$50-54$
$55-64$
$65-74$
$\geq 75$

Postherpetic neuralgia, ${ }^{\star} \%$ of herpes zoster cases

$50-54$
$55-64$
65

$\geq 75$

Case-fatality rate, $\% \dagger$

$50-54$
$55-64$
$65-74$
$\geq 75$

\section{Health care resource use}

Hospitalization, \% of herpes zoster cases

$50-54$
$55-64$
$65-74$
$\geq 75$

Consultations, per herpes zoster case

$50-54$
$55-64$
$65-74$
$\geq 75$

Length of hospital stay, $d$, mean

$50-54$
$55-64$
$65-74$
$\geq 75$

\section{Costs, $\ddagger$ in 2018 Can\$}

Herpes zoster-related hospitalization, per day

Herpes zoster-related consultations

Treatment per herpes zoster episode

Treatment per postherpetic neuralgia episode

\section{QALYs lost§}

\section{Herpes zoster}

$50-59$
$60-69$
$\geq 70$

Postherpetic neuralgia

$\begin{array}{llll}50-59 & 0.041 & 0.032^{31} & 0.052^{31} \\ 60-69 & 0.192 & 0.103^{31} & 0.290^{31} \\ \geq 70 & 0.234 & 0.191^{31} & 0.290^{31}\end{array}$

\begin{tabular}{|c|c|c|}
\hline 3.8 & $3.5^{25}$ & $4.2^{26}$ \\
\hline 6 & $5.1^{25}$ & $6.9^{26}$ \\
\hline 8.6 & $7.3^{25}$ & $10.0^{26}$ \\
\hline 9.9 & $8.0^{25}$ & $11.8^{26}$ \\
\hline 9.4 & $6.9^{21}$ & $11.9^{21}$ \\
\hline 9.4 & $6.9^{21}$ & $11.9^{21}$ \\
\hline 26 & $18.5^{21}$ & $33.4^{21}$ \\
\hline 27.7 & $22.0^{21}$ & $33.4^{21}$ \\
\hline 0 & $0.000^{21}$ & $0.002^{21}$ \\
\hline 0 & $0.000^{21}$ & $0.002^{21}$ \\
\hline 0.012 & $0.012^{21}$ & $0.083^{21}$ \\
\hline 0.076 & $0.040^{21}$ & $0.083^{21}$ \\
\hline
\end{tabular}
$1.6^{21}$ $2.5^{21}$ $5.1^{21}$ $15.6^{21}$

$12.7^{21}$

$14.7^{29}$

$16.5^{29}$

$23.7^{29}$

$\begin{array}{ccc}918^{30} & 495^{21} & 1483^{21} \\ 28^{30} & 24^{30} & 113^{29} \\ 136^{30} & 55^{21} & 255^{29} \\ 1588^{30} & 969^{30} & 2707^{21}\end{array}$

$\begin{array}{ccc}0.009 & 0.006^{31} & 0.012^{31} \\ 0.01 & 0.006^{31} & 0.013^{31} \\ 0.01 & 0.007^{31} & 0.014^{31} \\ & & \\ 0.041 & 0.032^{31} & 0.052^{31} \\ 0.192 & 0.103^{31} & 0.290^{31} \\ 0.234 & 0.191^{31} & 0.290^{31}\end{array}$

\section{References}

Brisson et al., ${ }^{21}$ Letellier et al., ${ }^{25}$ Russell et al. ${ }^{26}$ Tanuseputro et al.. ${ }^{27}$ Marra et al. ${ }^{28}$

Oxman et al., ${ }^{10}$ Brisson et al. ${ }^{21}$

Edmunds et al., ${ }^{4}$ Brisson et al. ${ }^{21}$

Brisson et al., ${ }^{3}$ Brisson et al., ${ }^{21}$ Letellier et al., ${ }^{25}$ Tanuseputro et al. ${ }^{27}$

Brisson et al., ${ }^{21}$ Letellier et al., ${ }^{25}$ Najafzadeh et al. ${ }^{29}$

Brisson et al., ${ }^{21}$ Letellier et al., ${ }^{25}$ Najafzadeh et al. ${ }^{29}$

Brisson et al., ${ }^{21}$ Najafzadeh et al., ${ }^{29}$ Friesen et al..$^{30}$

Brisson et al., ${ }^{21}$ Najafzadeh et al. ${ }^{29}$ Friesen et al. ${ }^{30}$

Brisson et al., ${ }^{21}$ Najafzadeh et al., ${ }^{29}$ Friesen et al. ${ }^{30}$

Brisson et al., ${ }^{21}$ Najafzadeh et al., ${ }^{29}$ Friesen et al. ${ }^{30}$

Drolet et al., ${ }^{11}$ Brisson et al., ${ }^{21}$ Brisson et al. ${ }^{31}$

Note: base value $=$ mean of minimum and maximum values identified in literature, maximum $=$ maximum values identified in literature, minimum $=$ minimum values identified in literature, QALY = quality-adjusted life-year.

*Postherpetic neuralgia was defined as clinically significant pain persisting for more than 90 days after onset of rash.

†Given the scarcity of data on herpes zoster-related mortality in Canada, we used case-fatality values estimated in a previous study in England and Wales. ${ }^{4}$

†Values from Friesen and colleagues ${ }^{30}$ were used as the base values.

$\S$ This variable captures, in a single measure, morbidity and mortality associated with a disease. Data for QALYs lost were obtained by measuring QALY-weight (or disutility), ranging from 0 to 1 , where a weight of 1 corresponds to optimal health and a weight of 0 corresponds to a health state judged as equivalent to death. The QALY lost per case is the difference in QALY weights with and without the disease, multiplied by duration of the disease. The QALY weights were taken from MASTER, a pan-Canadian, multicentre 6-month prospective study, which recruited patients aged $\geq 50$ years who presented with herpes zoster or postherpetic neuralgia, as described by Drolet and colleagues ${ }^{11}$ and Brisson and colleagues. ${ }^{31}$ Calculation of QALY lost is explained in detail by Brisson and colleagues. ${ }^{21}$ ILetellier and colleages ${ }^{25}$ did not present data by specific age groups, but we had access to the original data from Quebec administrative databases (2001 to 2015 ); for the purposes of our analysis, we estimated the incidence of herpes zoster by age groups. 


\section{Sensitivity analyses}

We performed a probability sensitivity analysis by assigning a triangular probability distribution to each parameter and then drawing 30000 combinations of these parameter values using Latin hypercube sampling. The minimum and maximum values of the distribution were the minimum and maximum value identified from the literature, and the median or mode is the base value presented in Table 1 . We present all model predictions as the median and $90 \%$ uncertainty interval (UI; the 5th and 95th percentiles taken from the distribution of 30000 simulation results).

We also performed univariable sensitivity analyses for the key model parameters (e.g., percentage of herpes zoster cases with development of postherpetic neuralgia, QALYs lost to postherpetic neuralgia). To do so, we fixed 1 key parameter value to its minimum or maximum value and varied all other parameters using the same probability distributions as for the main analysis (Table A4 in Appendix 1). In addition, we examined the potential impact of a single dose of the recombinant vaccine and of vaccination limited to immunocompetent adults (Table A2 in Appendix 1).

\section{Ethics approval}

For this modelling study, no ethics approval was required or obtained.

\section{Results}

In total, 90623 cases of herpes zoster, 13575 cases of ophthalmic herpes zoster and 17502 cases of postherpetic neuralgia were predicted to occur each year in Canada among adults aged 50 years or older (Table 2). Most of the burden of disease would occur in adults aged 70 years or older.

\section{Effectiveness of vaccination}

The NNV was higher for the live vaccine than for the recombinant vaccine for all herpes zoster-related events that we investigated (Table 3). The difference in NNV between the 2 vaccines increased with increasing age at vaccination, mainly because of the decline in vaccine efficacy by age with the live vaccine. For example, for adults exactly 60 years of age, the median NNV to prevent 1 case of herpes zoster was 18 (90\% UI 9-28) for the live vaccine versus 8 (90\% UI 6-21) for the recombinant vaccine, and the median NNV to prevent 1 case of postherpetic neuralgia was 78 (95\% UI 31-150) for the live vaccine versus 33 (90\% UI 23-128) for the recombinant vaccine. In contrast, for adults exactly 75 years of age, the median NNV for herpes zoster was 42 (90\% UI $32-63)$ for the live vaccine versus 11 (90\% UI 9-19) for the recombinant vaccine, whereas for postherpetic neuralgia, the median NNV was 78 (90\% UI 51-102) for the live vaccine versus 40 (90\% UI 31-71) for the recombinant vaccine.

\section{Cost-effectiveness}

Vaccinating adults aged 65 to 75 years against herpes zoster was predicted to result in cost-effectiveness ratios below $\$ 45000$ per QALY gained, for both vaccines and under all scenarios investigated (Figure 1; Table A3 in Appendix 1). However, there were considerable differences in cost-effectiveness ratios between the 2 vaccines. For the recombinant vaccine, the median costeffectiveness ratio predictions varied between cost-saving and \$25881 per QALY gained. Above 60 years, the cost-effectiveness ratios were relatively stable by age at vaccination, with the variability mainly due to vaccination cost. For the live vaccine, the median cost-effectiveness ratio predictions varied between costsaving and \$130587 per QALY gained. The cost-effectiveness ratios for the live vaccine were highly sensitive to age at vaccination, but remained below $\$ 45000$ per QALY gained for those between 65 and 75 years. Cost-effectiveness ratios were higher among adults older than 75 years, because of lower vaccine efficacy, and among adults younger than 65 years, because of waning vaccine efficacy (Figure A2 in Appendix 1).

Finally, the recombinant vaccine was estimated to be more cost-effective than the live vaccine for all ages at vaccination. We estimated that, depending on the age at vaccination, the cost for the complete series of recombinant vaccine could be $\$ 150$ to $\$ 200$ more than the live vaccine and still be considered costeffective using the threshold of $\$ 45000$ per QALY gained (Figure A4 in Appendix 1). The cost-effectiveness acceptability curves for the vaccination of adults aged 65 years indicated that for the recombinant vaccine, most of our simulations ( $>70 \%$ ) would be cost-effective for cost-effectiveness thresholds of $\$ 15000$ or more per QALY gained (assuming vaccine costs of $\$ 140$ or $\$ 200$ ). For the live vaccine, most of our simulations (> 70\%) would be cost-effective for cost-effectiveness thresholds of $\$ 30000$ or

Table 2: Yearly burden of illness in Canada*

\begin{tabular}{|c|c|c|c|c|}
\hline \multirow[b]{2}{*}{ Variable } & \multicolumn{3}{|c|}{ Age group; no. (\%) of population } & \multirow[b]{2}{*}{ Total, no. (90\% UI) } \\
\hline & $50-59$ yr & $60-69 \mathrm{yr}$ & $\geq 70 \mathrm{yr}$ & \\
\hline Ophthalmic herpes zoster & $3831(28)$ & $4355(32)$ & $5354(39)$ & 13575 (10 403-16 972) \\
\hline Postherpetic neuralgia & $2405(14)$ & $5398(31)$ & $9681(55)$ & 17502 (15 512-19 707) \\
\hline Death & $0(1)$ & $5(21)$ & $20(78)$ & $26(18-36)$ \\
\hline
\end{tabular}


Table 3: Estimated NNV with herpes zoster vaccines to prevent herpes zoster-related events, by age at vaccination

Type of vaccine; median NNV (90\% UI)

Herpes zoster-related event, by age at vaccination ${ }^{\star}$

$50 \mathrm{yr}$

Herpes zoster

Ophthalmic herpes zoster

Postherpetic neuralgia

Hospital admission

Death

$60 \mathrm{yr}$

Herpes zoster

Ophthalmic herpes zoster

Postherpetic neuralgia

Hospital admission

Death

$65 \mathrm{yr}$

Herpes zoster

Ophthalmic herpes zoster

Postherpetic neuralgia

Hospital admission

Death

$70 \mathrm{yr}$

Herpes zoster

Ophthalmic herpes zoster

Postherpetic neuralgia

Hospital admission

Death

$75 \mathrm{yr}$

Herpes zoster

Ophthalmic herpes zoster

Postherpetic neuralgia

Hospital admission

Death

$80 \mathrm{yr}$

Herpes zoster

Ophthalmic herpes zoster

Postherpetic neuralgia

Hospital admission

Death

\section{5 yr}

Herpes zoster

Ophthalmic herpes zoster

Postherpetic neuralgia

Hospital admission

Death
Live attenuated vaccine

$$
\begin{gathered}
15(6-28) \\
103(34-230) \\
106(26-341) \\
638(103-2419)
\end{gathered}
$$

71898 (13 407-2 610 325)

$$
18(9-28)
$$

$122(56-229)$

78 (31-150)

463 (119-1136)

39072 (14 670-91 623)

$$
\begin{gathered}
21(13-31) \\
138(76-285) \\
64(33-93) \\
335(124-700) \\
\hline 27828(14354-41590)
\end{gathered}
$$

$$
\begin{gathered}
28(21-43) \\
196(123-362) \\
73(42-96) \\
289(133-494) \\
32120(18131-43416)
\end{gathered}
$$

$$
42 \text { (32-63) }
$$$$
295(171-586)
$$$$
78 \text { (51-102) }
$$$$
215(126-378)
$$

34638 (21 718-50 059)

$$
\begin{gathered}
75(47-125) \\
\hline 523(257-1136) \\
97(68-126) \\
269(169-466) \\
43125(29410-61470)
\end{gathered}
$$

$$
\begin{gathered}
142(67-380) \\
983(377-3133) \\
124(94-164) \\
351(229-604) \\
55957(40079-79288)
\end{gathered}
$$

\section{Recombinant subunit vaccine}

$$
7 \text { (5-26) }
$$

49 (29-193)

36 (22-288)

171 (84-2061)

19473 (11 148-2 200338$)$

$$
\begin{gathered}
8(6-21) \\
54(34-150) \\
33(23-128) \\
149(84-947) \\
15915(10625-8621) \\
8(6-18) \\
57(37-138) \\
31(23-73) \\
137(82-613) \\
13672(9865-32620)
\end{gathered}
$$

$$
\begin{gathered}
9(7-19) \\
65(43-139) \\
35(27-73) \\
130(83-385) \\
15753(11630-32797)
\end{gathered}
$$

$$
11(9-19)
$$

77 (48-161)

$40(31-71)$

$116(75-226)$

18139 (13 206-32 667)

$14(11-22)$

$96(60-190)$

$50(40-80)$

$145(95-267)$

22811 (16 801-37 057)

$$
\begin{gathered}
18(15-25) \\
124(79-237) \\
66(52-94) \\
188(125-328)
\end{gathered}
$$

29816 (22 096-44 152)

Note: $90 \% \mathrm{UI}=$ uncertainty interval (based on 5th and 95th percentiles of 30000 simulation results), NNV = number needed to vaccinate. “Ages shown are individuals' exact age. 
more per QALY gained (assuming vaccine costs of $\$ 140$ ) and $\$ 50000$ or more per QALY gained (assuming vaccine costs of \$200) (Figure A3 in Appendix 1).

In the sensitivity analysis, the median cost-effectiveness ratios for the recombinant vaccine remained below the threshold of $\$ 45000$ per QALY gained for all scenarios investigated (Table A4, Table A5 and Figure A5 in Appendix 1). However, the median cost-effectiveness ratios for the live vaccine were highly sensitive to the parameters that determined the burden of herpes zoster and postherpetic neuralgia (e.g., incidence of herpes zoster, proportion of herpes zoster cases leading to postherpetic neuralgia and QALYs lost to postherpetic neuralgia). Of note, our results remained robust when we used discount rates of $0 \%$ and $5 \%$. The choice of discount rate has less impact for herpes zoster vaccines (relative to other vaccines) because the benefits accrue shortly after vaccination. Finally, when assuming that 2 doses were necessary for the recombinant vaccine to provide efficacy, our model predicted that the compliance with the second dose of the recombinant would have to be less than $50 \%$ to produce health benefits lower than using the live vaccine.

\section{Interpretation}

Our model predicted that the recombinant subunit zoster vaccine is likely cost-effective in Canada for adults 60 years or older and that it provides greater health benefits than the live attenuated zoster vaccine for all age groups. Thus, at a similar cost per series, the recombinant vaccine is likely a more cost-effective option than the live vaccine. The cost per series for the live vaccine would have to be $\$ 150$ to $\$ 200$ lower than for the recombinant vaccine for it to be considered a cost-effective alternative.

These results are consistent with other economic analyses of vaccination against herpes zoster conducted in the United States and the Netherlands, which predicted that vaccination with either vaccine is highly likely to be cost-effective, but at the same vaccine price, vaccination with the recombinant vaccine is more cost-effective. ${ }^{34-36}$

On the basis of the cost-effectiveness analysis and results presented here, $\mathrm{NACl}$ recommended that adults 50 years or older receive vaccination with the recombinant vaccine. ${ }^{19}$ Although that vaccine is predicted to be cost-effective for adults aged 60

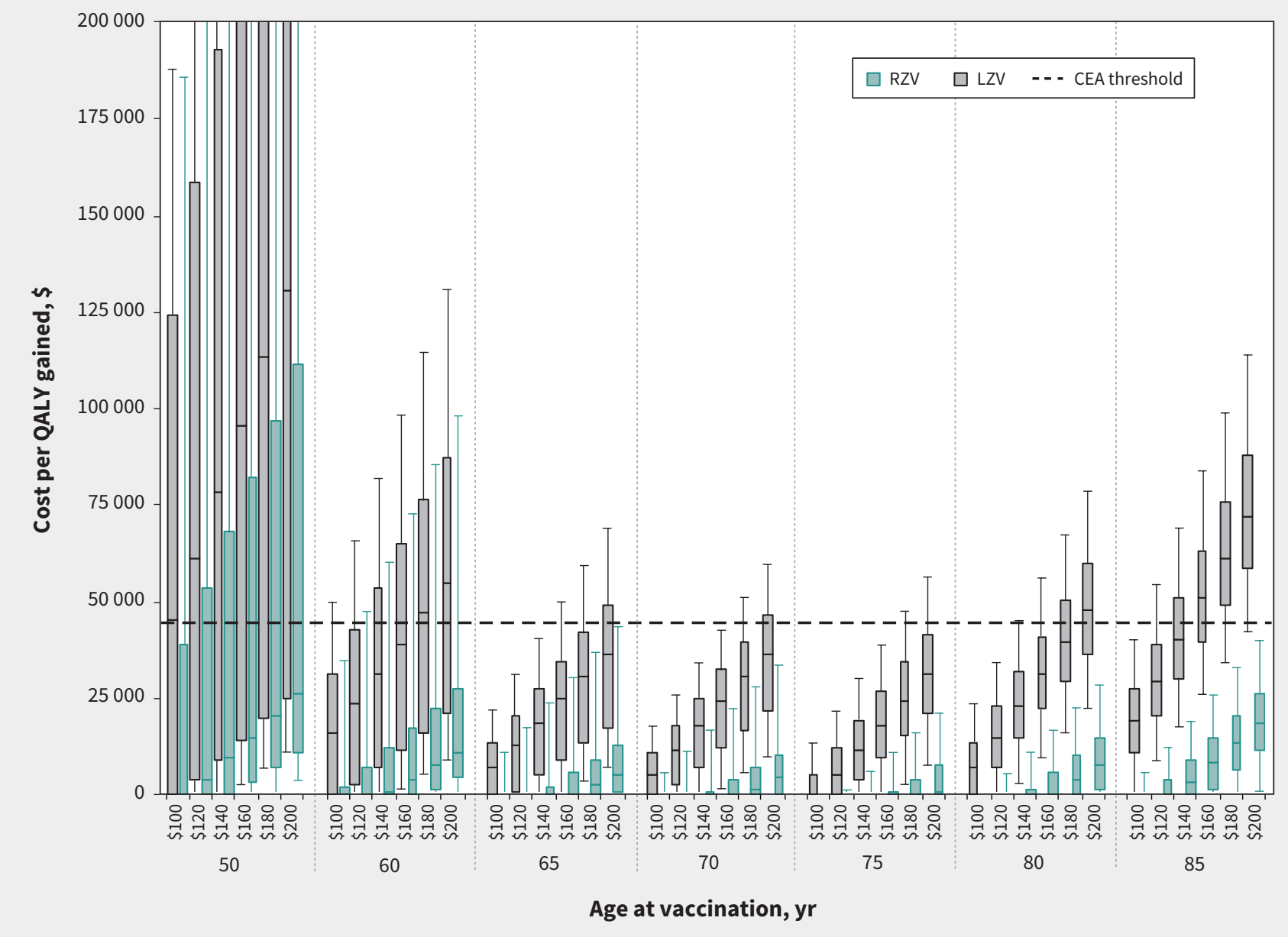

Figure 1: Cost per quality-adjusted life-year (QALY) gained of vaccination with the recombinant subunit zoster vaccine (RZV) and the live attenuated zoster vaccine (LZV) compared with no vaccination, by age at vaccination and vaccine cost (complete series). Box plots represent the 5 th, 25th, 50th, 75th and 95th percentiles from 30000 simulation results. Costs are reported in 2018 Canadian dollars. CEA = cost-effectiveness analysis. 
years or older, it may not be feasible to vaccinate all of these individuals. Hence, in accordance with our cost-effectiveness results, $\mathrm{NACl}$ indicated that, for publicly funded programs, vaccination of adults aged between 65 and 79 years would be the most cost-effective option.

Provincial immunization committees have made different recommendations in terms of the age cohorts to be targeted. The Comité d'immunisation du Québec recommended vaccination with the recombinant vaccine for adults aged 65 years or older, but noted that if it was not economically feasible to target all adults in this age group, adults 70 years or older should be prioritized, because of the greater incidence of herpes zoster and postherpetic neuralgia in this age group. ${ }^{20}$ Conversely, in Ontario, the live vaccine is publicly funded for adults aged 65 to 70 years, but physicians are obliged to offer both $\mathrm{NACl}$ recommended vaccines to their patients. ${ }^{37}$ In British Columbia, vaccination against herpes zoster is recommended for adults aged 50 years or older, but there is currently no publicly funded vaccination program. ${ }^{38}$

Our study had several strengths. It represents a unique examination of the effectiveness and cost-effectiveness of both the live and recombinant vaccines in a Canadian context, and our results are consistent with other economic analyses of herpes zoster vaccines from other counties. ${ }^{34-36,39}$ To capture the uncertainty around the duration of protection with herpes zoster vaccines, our predictions are based on simulations using 6 different functions for waning of vaccine efficacy (Figure A2 in Appendix 1). We have presented all predictions with $90 \%$ UIs, which captures the variability in the estimates of incidence and burden of disease of herpes zoster across Canada. Finally, the conclusions remained robust in our sensitivity analyses.

\section{Limitations}

The limitations of this study were mainly related to the availability of empiric data. First, a key factor influencing the costeffectiveness of both vaccines is the duration of protection. Although both trial and postlicensure studies suggest that the efficacy of the live vaccine declines substantially over time, ${ }^{16-18}$ there are no long-term efficacy data for the recombinant vaccine. We captured the uncertainty in the duration of the recombinant vaccine by means of $90 \%$ UIs and predicted that although waning can affect the cost-effectiveness ratio value, it does not affect the conclusion that this vaccine is likely cost-effective for adults aged 60 years or older.

There may be lower compliance with the second dose of the recombinant vaccine because of grade 3 adverse events described in the trial. ${ }^{14}$ There are reports from the US that some health care providers are deciding not to administer the second dose after observation of adverse effects following the first dose. ${ }^{40}$ We examined an extreme scenario in which there would be no vaccine efficacy for adults vaccinated with only 1 dose. Our model predicted that compliance with the second dose had to be less than $50 \%$ to produce health benefits lower than would be achieved using the live vaccine. Preliminary data from the US have suggested that compliance with the second dose is about $75 \%$ to $85 \%{ }^{41}$
The randomized trials assessing the efficacy of both vaccines were conducted in healthy, immunocompetent populations. Although some recent unpublished data have suggested that the recombinant vaccine may be slightly less effective against herpes zoster in immunosuppressed populations, ${ }^{42,43}$ there is no information on whether vaccine efficacy changes if vaccinated adults become immunosuppressed. In our study, we assumed that vaccine efficacy did not change among vaccinated adults who become immunosuppressed. This assumption could lead to overestimation of the effectiveness of herpes zoster vaccination, depending on the proportion of adults who become immunosuppressed over time and on the extent of the decline in vaccine efficacy after they become immunosuppressed.

\section{Conclusion}

Our modelling analysis suggests that vaccination against herpes zoster is most likely a cost-effective intervention in Canada. However, vaccination with the recombinant subunit zoster vaccine is predicted to provide greater effectiveness for all age groups and is likely to be more cost-effective than the live attenuated zoster vaccine. Future research should focus on assessing the long-term durability of 2 doses of the recombinant vaccine, compliance with the second dose and efficacy of a single dose of the vaccine.

\section{References}

1. Gnann JW Jr, Whitley RJ. Clinical practice. Herpes zoster. N Engl J Med 2002;347:340-6.

2. Head H, Campbell AW, Kennedy PG. The pathology of herpes zoster and its bearing on sensory localisation. Rev Med Virol 1997;7:131-43.

3. Brisson M, Edmunds WJ, Law B, et al. Epidemiology of varicella zoster virus infection in Canada and the United Kingdom [published erratum in Epidemiol Infect 2015;143:1332]. Epidemiol Infect 2001;127:305-14.

4. Edmunds WJ, Brisson M, Rose JD. The epidemiology of herpes zoster and potential cost-effectiveness of vaccination in England and Wales. Vaccine 2001;19:3076-90.

5. Insinga RP, Itzler RF, Pellissier JM, et al. The incidence of herpes zoster in a United States administrative database. J Gen Intern Med 2005;20:748-53.

6. Oxman MN. Clinical manifestation of herpes zoster. In: Arvin AM, Gerson AA, editors. Varicella zoster virus: virology and clinical management. Cambridge (UK): Cambridge University Press; 2000:246-75.

7. Opstelten W, Zuithoff NP, van Essen GA, et al. Predicting postherpetic neuralgia in elderly primary care patients with herpes zoster: prospective prognostic study. Pain 2007;132(Suppl 1):S52-9.

8. Schmader KE. Epidemiology and impact on quality of life of postherpetic neuralgia and painful diabetic neuropathy. Clin J Pain 2002;18:350-4.

9. Drolet M, Brisson M, Schmader K, et al. Predictors of postherpetic neuralgia among patients with herpes zoster: a prospective study. J Pain 2010;11:1211-21.

10. Oxman MN, Levin MJ, Johnson GR, et al. A vaccine to prevent herpes zoster and postherpetic neuralgia in older adults. N Engl J Med 2005;352:2271-84.

11. Drolet M, Brisson M, Schmader KE, et al. The impact of herpes zoster and postherpetic neuralgia on health-related quality of life: a prospective study. CMAJ 2010;182:1731-6.

12. Johnson RW, Wasner G, Saddier P, et al. Herpes zoster and postherpetic neuralgia: optimizing management in the elderly patient. Drugs Aging 2008;25:991-1006.

13. Drolet M, Oxman MN, Levin MJ, et al. Vaccination against herpes zoster in developed countries: state of the evidence. Hum Vaccin Immunother 2013;9:1177-84.

14. Lal H, Cunningham AL, Godeaux O, et al. Efficacy of an adjuvanted herpes zoster subunit vaccine in older adults. N Engl J Med 2015;372:2087-96.

15. Pauksens K, Volpe S, Schwarz T, et al. Persistence of immune response to an adjuvanted varicella-zoster virus subunit candidate vaccine for up to year 9 in older adults [poster]. IDSA ID Week; 2017 Oct. 4-8; San Diego.

16. Morrison VA, Johnson GR, Schmader KE, et al. Long-term persistence of zoster vaccine efficacy. Clin Infect Dis 2015;60:900-9. 
17. Tseng HF, Harpaz R, Luo Y, et al. Declining effectiveness of herpes zoster vaccine in adults aged $\geq 60$ years. $J$ Infect Dis 2016;213:1872-5.

18. Izurieta HS, Wernecke M, Kelman J, et al. Effectiveness and duration of protection provided by the live-attenuated herpes zoster vaccine in the Medicare population ages 65 years and older. Clin Infect Dis 2017;64:785-93.

19. Updated recommendations on the use of herpes zoster vaccines: an Advisory Committee Statement (ACS) National Advisory Committee on Immunization (NACI). Ottawa: Public Health Agency of Canada; 2018. Available: https://www.canada .ca/en/services/health/publications/healthy-living/updated-recommendations -use-herpes-zoster-vaccines.html (accessed 2019 Feb. 22).

20. Comité sur l'immunisation du Québec (CIQ). Avis sur la pertinence d'ajouter la vaccination contre le zona au Programme québécois d'immunisation. Québec (QC): Institut national de santé publique du Québec; 2018. Available: https:// www.inspq.qc.ca/sites/default/files/publications/2381_pertinence_vaccination _zona_programme_quebecois_immunisation.pdf (accessed 2019 Feb. 22).

21. Brisson M, Pellissier JM, Camden S, et al. The potential cost-effectiveness of vaccination against herpes zoster and post-herpetic neuralgia. Hum Vaccin 2008;4:238-45.

22. Schmader KE, Oxman MN, Levin MJ, et al. Persistence of the efficacy of zoster vaccine in the shingles prevention study and the short-term persistence substudy. Clin Infect Dis 2012;55:1320-8.

23. Cunningham AL, Lal H, Kovac M, et al. Efficacy of the herpes zoster subunit vaccine in adults 70 years of age or older. N Engl J Med 2016;375:1019-32.

24. Bilcke J, Ogunjimi B, Hulstaert F, et al. Estimating the age-specific duration of herpes zoster vaccine protection: a matter of model choice? Vaccine 2012;30:2795-800.

25. Letellier MC, Amini R, Gilca V, et al. Herpes zoster burden in Canadian provinces: a narrative review and comparison with Quebec provincial data. Can J Infect Dis Med Microbiol 2018;2018:3285327.

26. Russell ML, Dover DC, Simmonds KA, et al. Shingles in Alberta: before and after publicly funded varicella vaccination. Vaccine 2014;32:6319-24.

27. Tanuseputro P, Zagorski B, Chan KJ, et al. Population-based incidence of herpes zoster after introduction of a publicly funded varicella vaccination program. Vaccine 2011;29:8580-4.

28. Marra F, Chong M, Najafzadeh M. Increasing incidence associated with herpes zoster infection in British Columbia, Canada. BMC Infect Dis 2016;16:589.

29. Najafzadeh M, Marra CA, Galanis E, et al. Cost effectiveness of herpes zoster vaccine in Canada. Pharmacoeconomics 2009;27:991-1004.

30. Friesen KJ, Chateau D, Falk J, et al. Cost of shingles: population-based burden of disease analysis of herpes zoster and postherpetic neuralgia. BMC Infect Dis 2017;17:69.

31. Brisson M, Johnson RW, Levin MJ, et al. Measuring herpes zoster (HZ) and postherpetic neuralgia (PHN) associated burden of illness, health care utilization and costs in Canada: a clinical epidemiological study. Can J Infect Dis Med Microbiol 2006;17:381.

32. Consumer price index, annual average, not seasonally adjusted. Ottawa: Statistics Canada; 2019. Available: https://www150.statcan.gc.ca/t1/tbl1/en/tv.action ?pid=1810000501 (accessed 2019 May).

33. Cost-effectivess and strategic planning (WHO-CHOICE) . Geneva: World Health Organization; 2014. Available: http://www.who.int/choice/en (accessed 2019 Mar. 6).

34. Curran D, Patterson B, Varghese L, et al. Cost-effectiveness of an adjuvanted recombinant zoster vaccine in older adults in the United States. Vaccine 2018;36:5037-45.

35. Le $\mathrm{P}$, Rothberg MB. Cost-effectiveness of the adjuvanted herpes zoster subunit vaccine in older adults. JAMA Intern Med 2018;178:248-58.

36. de Boer PT, van Lier A, de Melker $\mathrm{H}$, et al. Cost-effectiveness of vaccination of immunocompetent older adults against herpes zoster in the Netherlands: a comparison between the adjuvanted subunit and live-attenuated vaccines. BMC Med 2018;16:228.

37. Immunization update: $\mathrm{NACl}$ recommends RZV (Shingrix ${ }^{\circledR}$ ) vaccine in persons aged 50 years and older. Ottawa: Ottawa Public Health; 2018. Available: https://www. ottawapublichealth.ca/Modules/News/blogcomments.aspx?feedld=9fd17063-0b6e -449b-8679-d24788af5476\&lang=en\&Blogld=ed6092fb-2df9-49f6-a711-978f84f3a2dc (accessed 2019 Mar. 7).

38. Shingles. Vancouver: BC Centre for Disease Control. Available: http://www. bccdc.ca/health-info/diseases-conditions/shingles (accessed 2019 Mar. 7).

39. Prosser LA, Harpaz R, Rose AM, et al. A cost-effectiveness analysis of vaccination for prevention of herpes zoster and related complications: input for national recommendations. Ann Intern Med 2019 Feb. 19. doi: 10.7326/M18-2347. [Epub ahead of print].

40. Hesse EM, Shimabukuro TT, Su JR, et al. Postlicensure safety surveillance of recombinant zoster vaccine (Shingrix) - United States, October 2017-June 2018. MMWR Morb Mortal Wkly Rep 2019;68:91-4.

41. Dooling K. Herpes zoster vaccines: update. Meeting of Advisory Committee on Immunization Practices; 2019 Feb. 27-28; Atlanta.

42. New data supports the safety and efficacy of GSK's Shingrix in preventing shingles in autologous haematopoietic stem cell transplant patients [media release]. London: GlaxoSmithKline; 2017 Dec. 6. Available: https://www.gsk. com/en-gb/media/press-releases/new-data-supports-the-safety-and-efficacy-of -gsk-s-shingrix-in-preventing-shingles-in-autologous-haematopoietic-stem-cell -transplant-patients/ (accessed 2019 Feb. 27).

43. Dagnew A, Ilhan O, Lee WS, et al. Immunogenicity, safety and post-hoc efficacy assessment of the adjuvanted recombinant zoster vaccine in adults with hematologic malignancies: a phase 3, randomized clinical trial [abstract]. IDSA ID Week; 2018 Oct. 3-7; San Francisco.
Competing interests: Philippe DeWals has received research grants and reimbursement for travel expenses from vaccine manufacturers, including the GSK group of companies, Novartis, Pfizer and Sanofi Pasteur. The quality-adjusted life-year estimates were partially derived from MASTER, a study conducted in 2005-2006 and funded by Merck Frosst Canada Ltd. through a collaborative research agreement between Merck and the study's scientific steering committee, of which Marc Brisson was a member. No other competing interests were declared.

This article has been peer reviewed.

Affiliations: Centre de recherche du Centre hospitalier de l'Université de Québec (Drolet, Zhou, Sauvageau, Gilca, Bénard, Brisson) and Département de médecine sociale et préventive (Sauvageau, DeWals, Bénard, Brisson), Université Laval; Institut national de santé publique du Québec (Sauvageau, DeWals, Gilca, Amini), Québec, Que.

Contributors: Mélanie Drolet and Marc Brisson were primarily responsible for the conception and design of the study, the acquisition of data, and the analysis and interpretation of results; they also drafted the first version of the manuscript. Zhou Zhou performed the analysis and revised the paper for important intellectual content. Chantal Sauvageau, Philippe DeWals, Vladimir Gilca, Rachid Amini and Élodie Bénard contributed to either the acquisition of the data or the analysis, and critically revised the paper for important intellectual content. All of the authors provided final approval of the version to be published, and agreed to be accountable for all aspects of the work.

Funding: This work was funded by the Public Health Agency of Canada, the Ministère de la Santé et des Services Sociaux du Québec, the Canadian Institutes of Health Research (Foundation scheme grant FDN143283) and the Fonds de recherche du Québec - Santé (support to Marc Brisson). The funders had no role in the study design, data collection, data analysis and interpretation, or the writing of this article.

Data sharing: The data available from this modelling study are presented in the tables and appendices of this article.

Accepted: July 3, 2019

Correspondence to: Marc Brisson, Marc.Brisson@crchudequebec. ulaval.ca 